\title{
Efeitos iniciais da reposição volêmica com solução salina hipertônica a 7,5\% na perfusão e oxigenação esplâncnica após choque hemorrágico ${ }^{1}$
}

\author{
Early effects of 7.5\% hypertonic saline solution on splanchnic perfusion after \\ hemorrhagic shock
}

\author{
Ruy Jorge Cruz Junior², Margareth M. Yada-Langui ${ }^{3}$, Alejandra Gallardo Garrido ${ }^{3}$, Luiz Francisco Poli de Figueiredo ${ }^{4}$, \\ Mauricio Rocha e Silva ${ }^{5}$ \\ 1. Trabalho realizado no Serviço de Fisiologia Aplicada - Instituto do Coração (InCor) da Faculdade de Medicina da Universidade de São \\ Paulo (FMUSP), Brasil. \\ 2. Médico Assistente, Doutor do InCor da FMUSP, Brasil. \\ 3. Pesquisadora, Doutora do InCor da FMUSP, Brasil. \\ 4. Professor Titular do Depto. de Cirurgia da UNIFESP-EPM, Professor Associado do Depto. de Cardiopneumologia da FMUSP, Brasil. \\ 5. Professor Titular do Depto. de Cardiopneumologia da FMUSP, Brasil.
}

\section{RESUMO}

Objetivo: Avaliar os efeitos hemodinâmicos sistêmicos e esplâncnico da expansão volêmica inicial com SSH em modelo de choque hemorrágico controlado. Métodos: Dez cães foram submetidos a sangramento controlado (20 ml/min) até uma pressão arterial média de $40 \pm 5$ mmHg (PAM). Após 30 minutos de choque, receberam 4 ml/Kg de SSH em 5 minutos e posteriormente observados sem intervenções adicionais durante 60 minutos. As variáveis hemodinâmicas sistêmicas foram obtidas de um cateter arterial e de um cateter de Swan-Ganz, enquanto as regionais através da cateterização da veia porta, fluxômetro ultrassônico na veia porta e um tonômetro na cavidade. A oferta, taxa de extração e consumo esplâncnico de oxigênio, $\mathrm{pH}$ intramucoso e os gradientes veno-arterial, porta-arterial e mucosa-arterial da $\mathrm{pCO}_{2}\left(\mathrm{D}_{\mathrm{ap}-\mathrm{a}} \mathrm{pCO}_{2}, \mathrm{D}_{\mathrm{vp-a}} \mathrm{pCO}_{2}\right.$ e $\mathrm{D}_{\mathrm{t}-\mathrm{a}} \mathrm{pCO}_{2}$, respectivamente), foram calculados. Resultados: A hemorragia (29,8 $22,4 \mathrm{ml} / \mathrm{Kg}$ ) reduziu a pressão arterial média (125 \pm 6 para $42 \pm 1 \mathrm{mmHg})$, o DC (1,9 $\pm 0,2$ para $0,6 \pm 0,1 \mathrm{~L} / \mathrm{min}$ ) e o fluxo porta ( $504 \pm 73$ para $126 \pm 12 \mathrm{ml} / \mathrm{min}$ ), enquanto elevou o $\mathrm{D}_{\text {ap-a }} \mathrm{pCO}_{2}(5,3 \pm 0,8$ para 19,9 $\pm 1,6 \mathrm{mmHg}), \mathrm{D}_{\mathrm{vp}-\mathrm{a}} \mathrm{pCO}_{2}(5,4 \pm 1,4$ para $22,6 \pm 2,1 \mathrm{mmHg})$ e o $\mathrm{D}_{\mathrm{t}-\mathrm{a}} \mathrm{pCO}_{2}(6,1 \pm 1,1 \mathrm{para} 43,8 \pm 7,5$ $\mathrm{mmHg}$ ). A infusão de SSH resultou em recuperação parcial dos fluxos sistêmico e porta. Atenuou os gradientes de $\mathrm{CO}_{2} \mathrm{com}^{-1}$ menor impacto sobre o $\mathrm{D}_{\mathrm{t}-\mathrm{a}} \mathrm{pCO}_{2}$. Conclusão: A SSH promoveu benefícios parciais na perfusão sistêmica e esplâncnica, os quais foram especialmente limitados na microcirculação regional, como demonstrado pelo $\mathrm{D}_{\mathrm{t}-\mathrm{a}} \mathrm{pCO}_{2}$. Além disso, as variáveis sistêmicas e regionais dependentes de oxigênio, não refletem a adequação da perfusão da mucosa gástrica, enfatizando a importância da monitorização deste território - pela tonometria - durante os estados de choque.

Descritores: Choque.Solução Salina Hipertônica. Hemorragia. Circulação Esplâncnica.

\begin{abstract}
Purpose: To evaluate the effects of SSH resuscitation on systemic and splanchnic hemodynamic variables in an experimental model of controlled hemorrhagic shock. Methods: Ten mongrel dogs were bled $(20 \mathrm{ml} / \mathrm{min})$ to a target mean arterial pressure (MAP) of 40 $\pm 5 \mathrm{mmHg}$. After 30 minutes of shock, animals received SSH infused in 5-minute and they were observed for 60 minutes thereafter. Systemic hemodynamics were evaluated through a Swan-Ganz and arterial catheters while gastrointestinal tract perfusion by a catheter inside the portal vein, an ultrasonic flowprobe around portal vein blood flow (PVBF) and a gastric tonometer. Splanchnic oxygen delivery and consumption, intramucosal $\mathrm{pH}$ and veno-arterial, portal-arterial and mucosal-arterial $\mathrm{pCO}_{2}$-gradients $\left(\mathrm{D}_{\mathrm{ap}-\mathrm{a}} \mathrm{pCO}_{2}, \mathrm{D}_{\mathrm{vp}-\mathrm{a}} \mathrm{pCO}_{2}\right.$ e $\mathrm{D}_{\mathrm{t}-\mathrm{a}} \mathrm{pCO}_{2}$, respectively) were assessed. Results: Hemorrhage $(29.8 \pm 2.4 \mathrm{ml} / \mathrm{Kg}$ ) induced significant decreases in MAP (125 \pm 6 to $42 \pm 1 \mathrm{mmHg})$, in CO $(1.9 \pm 0.2$ to $0.6 \pm 0.1 \mathrm{~L} / \mathrm{min})$, and PVBF (504 \pm 73 to $126 \pm 12 \mathrm{ml} / \mathrm{min}$ ) while significant increases were detected in $\mathrm{D}_{\text {ap-a }} \mathrm{pCO}_{2}(5.3 \pm 0.8$ to $19.9 \pm 1.6 \mathrm{mmHg}) \mathrm{D}_{\text {vp-a }} \mathrm{pCO}_{2}$ (5.4 \pm 1.4 to $22.6 \pm 2.1 \mathrm{mmHg}$ ) and $\mathrm{D}_{\mathrm{t}-\mathrm{a}} \mathrm{pCO}_{2}(6.1 \pm 1.1$ to $43.8 \pm 7.5 \mathrm{mmHg})$. SSH infusion promoted only partial benefits in systemic and splanchnic blood flows. Reduced $\mathrm{pCO}_{2}$ gradients but fewer effects in $\mathrm{D}_{\mathrm{t}-\mathrm{a}} \mathrm{pCO}_{2}$ were observed. Conclusion: The SSH infusion promoted partial systemic and splanchnic hemodynamic benefits. Those benefits were especially poor at the splanchnic microcirculation, as evaluated by $\mathrm{D}_{\mathrm{t}-\mathrm{a}} \mathrm{pCO}_{2}$. In addition, systemic and regional oxygen-derived variables do not reflect the regional microcirculation disturbances. Gastrointestinal tonometry clearly represents a useful tool for monitoring splanchnic perfusion in patients in hemodynamic shock.
\end{abstract}

Key words: Shock. Saline Solution, Hypertonic. Hemorrhage. Splanchnic Circulation. 


\section{Introdução}

O rápido controle do sangramento e a expansão volêmica adequada são intervenções fundamentais no manejo do choque hemorrágico, cuja finalidade é o restabelecimento da oxigenação e perfusão tecidual ${ }^{1}$. Tais intervenções podem reduzir significativamente a morbimortalidade em vítimas de trauma e em pacientes submetidos a cirurgias de grande porte ${ }^{1}$. Entretanto, tem sido demonstrado que apenas a restauração dos parâmetros hemodinâmicos sistêmicos e de transporte de oxigênio, comunente utilizados na prática clinica, não asseguram a adequação da perfusão no território esplâncnico após episódio de hemorragia ${ }^{2-4}$. No choque hemorrágico, assim como em quadros de choque de qualquer etiologia, a hipoperfusão do território esplâncnico, particularmente da mucosa gastrointestinal é de instalação precoce e desproporcional ao comprometimento sistêmico ${ }^{1-5}$. Tal fenômeno tem sido implicado na gênese, amplificação e perpetuação da resposta inflamatória sistêmica, e pode contribuir para o desenvolvimento da sepse e progressão da disfunção de múltiplos órgãos e sistemas ${ }^{1,3,6}$. Atualmente, a tonometria gástrica ou intestinal tem sido proposta como um método simples e pouco invasivo para avaliar a perfusão regional à beira-leito ${ }^{5-7}$. Este método possibilita a mensuração indireta da pressão parcial de $\mathrm{CO}_{2}$ na mucosa. Como a concentração deste gás nos tecidos tem relação inversa com fluxo, poder-se-ia inferir o fluxo para esta região utilizando esta metodologia ${ }^{3,7}$. Diversos autores têm utilizado a tonometria como marcador prognóstico e como guia terapêutico para otimização das manobras de ressuscitação em pacientes graves, mais especificamente, em vítimas de trauma, no choque séptico, no choque cardiogênico e cirurgias de grande porte incluindo transplantes e cirurgia cardíaca ${ }^{8,9}$. Embora os cristalóides sejam considerados os fluidos de primeira linha para a expansão volêmica, é crescente a evidência de que as características dos fluidos utilizados na ressuscitação volêmica de pacientes em choque pode afetar o curso da doença ${ }^{10}$. Os fluídos podem ter diferentes efeitos na resposta imune, ativação neutrofílica, lesão tecidual pós-ressuscitação, instalação e progressão da disfunção orgânica ${ }^{10}$. O Ringer lactato no choque hemorrágico tem sido associado a uma excessiva ativação neutrofílica e aumento da expressão de moléculas de adesão em neutrófilos e endotélio. Adicionalmente, o Ringer lactato têm sido associado à expressão de genes leucocitários envolvidos na inflamação, migração celular, apoptose, produção de citocinas e na resposta ao estresse ${ }^{10}$. Em contrapartida, a solução salina hipertônica suprime a atividade neutrofílica oxidativa e atenua a interação entre leucócitos, plaquetas e endotélio ${ }^{11}$. A menor interação leucócito-endotélio tem sido associada a redução do colapso microvascular, melhora da permeabilidade da parede vascular e melhora do fluxo na microcirculação ${ }^{11}$. Finalmente, o uso de cristalóides isotônicos está associado ao aumento do conteúdo de água nos tecidos sendo este mais acentuado no miocárdio e no trato digestivo, podendo afetar adversamente a perfusão regional podendo levar ao desenvolvimento da síndrome compartimental abdominal ${ }^{1}$. A solução salina hipertônica a 7,5\% (SSH) pode ser utilizada como fluido inicial na reposição volêmica no choque hemorrágico ${ }^{11}$. A experiência clínica disponível com esta solução sugere benefícios no tratamento da hipotensão póstraumática, principalmente nos traumas penetrantes e em vítimas de trauma craniano ${ }^{11}$. Muitos efeitos benéficos da SSH estão associados à rápida expansão do volume plasmático, às custas da transferência de líquido do compartimento intracelular para os compartimentos intersticial e intravascular ${ }^{11}$. Neste estudo, tivemos como objetivo avaliar os efeitos iniciais da reposição volêmica com solução salina hipertônica a 7,5\% na perfusão do território esplâncnico de cães submetidos a um modelo de choque hemorrágico controlado.

\section{Métodos}

Este projeto foi realizado em conformidade com as normas internacionais para utilização de animais de experimentação.

\section{Preparação animal}

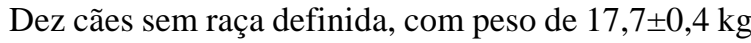
foram submetidos a um período de jejum de 12 horas com livre acesso a água. A anestesia realizada com 1,5 mg de sulfato de morfina por via intramuscular, seguido de $25 \mathrm{mg} /$ $\mathrm{Kg}$ de pentobarbital por via endovenosa. Doses adicionais de pentobarbital sódico ( $2 \mathrm{mg} / \mathrm{Kg}$ ) foram utilizadas quando necessário para manutenção do plano anestésico. Após entubação orotraqueal, os animais foram submetidos à ventilação mecânica (ventilador Takaoka modelo 670, São Paulo, SP, Brasil). Foi utilizado 1,0 de fração inspirada de oxigênio e a ventilação foi ajustada para obtenção de uma $\mathrm{pCO}_{2}$ arterial inicial de $40 \pm 5 \mathrm{mmHg}$. Após a cateterização vesical, os animais foram posicionados em decúbito dorsal sobre colchão térmico com a finalidade de manter a normotermia durante todo o procedimento. Através de uma inguinotomia direita, foram expostas a artéria e a veia femoral, sendo posicionados cateteres de politelieno (PE240), no interior da aorta abdominal e veia cava inferior, respectivamente. Após a obtenção do acesso venoso, foi realizada a infusão de ranitidina (25 mg) diluída em $50 \mathrm{ml}$ de solução fisiológica. O cateter de polietileno posicionado no interior da aorta abdominal foi utilizado para medida da pressão arterial média (PAM) e obtenção de sangue arterial para análise gasométrica e medidas do $\mathrm{pH}$, bicarbonato, déficit de bases, hematócrito, hemoglobina, sódio e cloro. A artéria femoral esquerda foi dissecada e cateterizada para a realização do modelo de choque hemorragico. Através da veia jugular externa direita, foi introduzido cateter de SwanGanz ${ }^{\text {9̀ }}$ A-131H-7F (Baxter Health Care Corporation, Irvine, CA - EUA), cuja extremidade distal foi posicionada na artéria pulmonar, por meio da análise das curvas de pressão. Este cateter foi conectado ao monitor de débito cardíaco (Vigilance, Baxter Edwards Critical Care, Irvine, CA - EUA), para medida da pressão média da artéria pulmonar, débito cardíaco e obtenção do sangue venoso misto. As medidas pressóricas foram realizadas através da conexão dos respectivos cateteres a transdutores de pressão (Transpac Disposable Transducer, Abbott, Chicago, IL - EUA) ligados em um sistema de aquisição de dados biológicos (modelo MP 100, Biopac System Inc., Goleta, CA - EUA) sendo os 
dados registrados em um computador através de software específico (ACqKnowledge ${ }^{\circledR}$ ?III MP 100 WSW).

$\mathrm{O}$ acesso à cavidade abdominal foi obtido por incisão mediana. Em seguida foi realizada a esplenectomia e através da veia esplênica foi posicionado um cateter de polietileno multiperfurado, no interior da veia porta, para a obtenção de amostras sanguíneas do território esplâncnico. Procedeu-se então a dissecção do ligamento hepatoduodenal com identificação e isolamento da veia porta, para o posicionamento de um fluxômetro ultra-sônico ao redor deste vaso (T206 small animal blood flowmeter - Transonic Systems Inc., Nova York - EUA) para a medida contínua do fluxo sangüíneo portal (FSVP).

Uma sonda de Levine (16F), foi introduzida por via oral e sua extremidade distal posicionada no interior da cavidade gástrica, para a lavagem do estomago com solução fisiológica aquecida até a remoção completa do resíduo alimentar. Após a retirada da sonda de Levine, foi introduzido pela cavidade oral o cateter TRIP ${ }^{\circledR}$ Tonometric Catheter 16F (Tonometrics Division, Helsinque - Finlândia) sendo este posicionado na região do antro gástrico, próximo a grande curvatura. Este cateter foi conectado ao sistema de leitura TONOCAP ${ }^{\circledR}$ (Datex-Engstrom Division, Helsinque Finlândida), permitindo a medida da pressão parcial de dióxido de carbono da mucosa gástrica $\left(\mathrm{prCO}_{2}\right)$.

\section{Protocolo experimental}

Trinta minutos após o final da preparação, foram obtidas as medidas basais (BL). Em seguida foi realizado protocolo de hemorragia controlada através de bomba perfusão automática (Harvard Apparatus, Massachusetts EUA), com velocidade de sangramento fixa em $20 \mathrm{ml} / \mathrm{min}$ até que a pressão arterial média atingisse valores de 40 $\mathrm{mmHg}(\mathrm{CH} 0)$. A PAM foi mantida nestes valores por mais 30 minutos (CH30), pela retirada ou infusão de sangue.

Após estes trinta minutos de choque ( $\mathrm{CHO}$ a $\mathrm{CH} 30$ ), foi iniciada a infusão de solução salina hipertônica a 7,5\% ( $4 \mathrm{ml} / \mathrm{kg}$ de peso em 5 minutos). Os animais foram observados por um período de uma hora após o início do tratamento (T0 a T60).

\section{Variáveis analisadas}

As pressões arterial média e da artéria pulmonar, e o fluxo sangüíneo da veia porta foram avaliados continuamente. O débito cardíaco (DC) foi determinado pela técnica de termodiluição, utilizando injeção em bolus de $3 \mathrm{ml}$ de solução salina isotônica a $20^{\circ} \mathrm{C}$, a cada 10 minutos. $\mathrm{O}$ déficit de bases, $\mathrm{pH}, \mathrm{pCO}_{2}, \mathrm{pO}_{2}$, saturação de hemoglobina, hematócrito, e o bicarbonato nas amostras de sangue proveniente da aorta abdominal, artéria pulmonar e veia porta foram obtidos nos momentos basais (BL), no período final do choque (CH30), e 20 e 60 minutos após o início do tratamento (T20 e T60, respectivamente). As amostras de sangue foram analisadas imediatamente após a sua coleta pelo analisador de gases Stat Profile Ultra Analyzer (Nova Biomedical, Waltham, MA, EUA). O sódio e cloro foram mensurados nos mesmos momentos experimentais (BL, CH30, T20 e T60) no sangue obtido através da artéria femoral.
$\mathrm{O} \mathrm{pCO}_{2}$ da mucosa gástrica $\left(\mathrm{prCO}_{2}\right)$ foi avaliado automaticamente a cada 20 minutos. $\mathrm{O}$ pH intramucoso gástrico (pHi) foi determinado de acordo com o método de Fiddian-Green et al ${ }^{12}$, usando uma modificação da equação de Henderson-Hasselbach: $\mathrm{pHi}=6,1+\log \left(\left[\mathrm{HCO}_{3}^{-}\right] / \mathrm{prCO}_{2} \mathrm{x}\right.$ 0,031), onde $\mathrm{HCO}_{3}$ - é a concentração arterial de bicarbonato $(\mathrm{mmol} / \mathrm{L})$ e $\mathrm{prCO}_{2}$ representa a medida da pressão parcial de dióxido de carbono obtido através da tonometria.

A oferta, consumo e taxa de extração sistêmica e regional de oxigênio $\left(\mathrm{DO}_{2}\right.$ sist, $\mathrm{VO}_{2}$ sist, $\mathrm{TEO}_{2}$ sist, $\mathrm{DO}_{2}$ esplanc, $\mathrm{VO}_{2}$ esplanc e $\mathrm{TEO}_{2}$ esplanc respectivamente) foram calculadas utilizando fórmulas padrão. Três gradientes da pressão parcial de dióxido de carbono $\left(\mathrm{pCO}_{2}\right)$ foram calculados: (1) diferença entre a $\mathrm{pCO}_{2}$ da veia porta e o sangue arterial, (2) diferença da $\mathrm{pCO}_{2}$ da artéria pulmonar e do sangue arterial e (3) diferença da $\mathrm{pCO}_{2}$ obtida tonometricamente (mucosa intestinal) e a $\mathrm{pCO}_{2}$ do sangue arterial, ( $\mathrm{D}_{\mathrm{vp}-\mathrm{a}} \mathrm{pCO}_{2}, \mathrm{D}_{\mathrm{ap}-\mathrm{a}} \mathrm{pCO}_{2} \mathrm{e}_{\mathrm{t}-\mathrm{a}} \mathrm{pCO}_{2}$, respectivamente).

\section{Metodologia estatística}

Os resultados são apresentados como média \pm erro padrão. Análise estatística foi realizada usando o software Statistic Package for Social Sciences for Windows (versão 6,0 - SPSS Inc., Chicago, IL, EUA). Foi utilizada análise de variância controlada pelo teste de Tukey. Para análise da correlação entre as variáveis foi utilizado o teste de Spearman. Diferença estatística foi considerada para valores de $p$ menores que 0,05 .

\section{Resultados}

O protocolo de hemorragia controlada, com a perda de aproximadamente $43 \%$ do volume sangüíneo total (29,8 \pm 2,4 ml/Kg), induziu uma redução significativa da pressão arterial média, do débito cardíaco e da saturação venosa mista de oxigênio (Figura 1, Tabela 1).

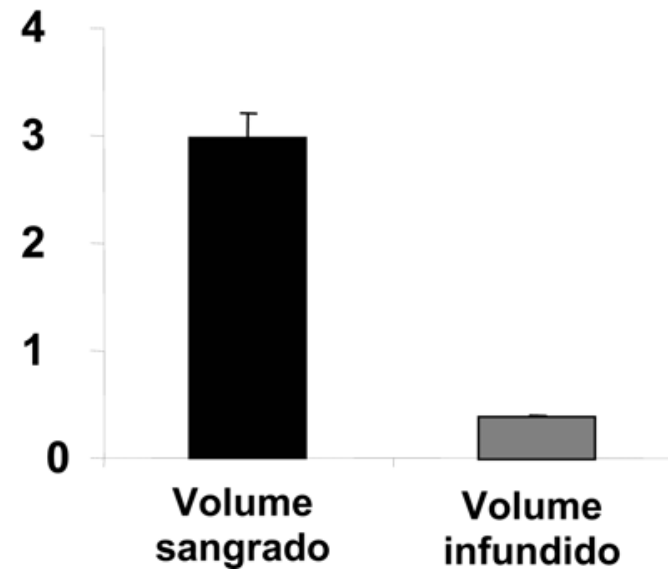

FIGURA 1 - Volume sangüíneo total sangrado e volume de SSH infundido. 
TABELA 1 - Variáveis hemodinâmicas e laboratoriais sistêmicas durante o protocolo experimental

\begin{tabular}{lllll}
\hline & BL & CH30 & T20 & T60 \\
\hline DC $(\mathbf{L} / \mathbf{m i n})$ & $1,9 \pm 0,2$ & $0,6 \pm 0,1^{*}$ & $1,1 \pm 0,1^{*}$ & $0,9 \pm 0,1^{*}$ \\
PAM $(\mathbf{m m H g})$ & $125,5 \pm 6,8$ & $42 \pm 1,5^{*}$ & $76,6 \pm 2,9^{*}$ & $88,8 \pm 3,3^{*}$ \\
PMAP $(\mathbf{m m H g})$ & $16,4 \pm 1,5$ & $8,9 \pm 1,4^{*}$ & $10,3 \pm 1,4^{*}$ & $9,7 \pm 1,4^{*}$ \\
SvO2 $(\%)$ & $84,3 \pm 1,2$ & $25,4 \pm 3,9^{*}$ & $58,5 \pm 3,7^{*}$ & $52,7 \pm 4,3^{*}$ \\
Hemoglobina arterial $(\%)$ & $14,9 \pm 0,3$ & $12,4 \pm 0,4^{*}$ & $9,9 \pm 0,4^{*}$ & $10,8 \pm 0,4^{*}$ \\
Lactato arterial $(\boldsymbol{\mu} \mathbf{m o l} / \mathbf{L})$ & $0,8 \pm 0,2$ & $4,6 \pm 0,8^{*}$ & $4,8 \pm 0,7^{*}$ & $3,7 \pm 0,5^{*}$ \\
Excesso de bases arterial $(\mathbf{m m o l} / \mathbf{L})$ & $-0,6 \pm 0,6$ & $-6,5 \pm 0,7^{*}$ & $-6,8 \pm 0,9^{*}$ & $-5,8 \pm 0,8^{*}$ \\
Na+ arterial $(\mathbf{m m o l} / \mathbf{L})$ & $145,3 \pm 0,5$ & $144,7 \pm 0,7$ & $156,1 \pm 0,7^{*}$ & $154,2 \pm 0,8^{*}$ \\
Cl- arterial $(\mathbf{m m o l} / \mathbf{L})$ & $109 \pm 2$ & $110 \pm 3$ & $128 \pm 2,4^{*}$ & $126 \pm 5^{*}$ \\
\hline
\end{tabular}

DC: débito cardíaco, PAM: pressão média arterial, PMAP: pressão média da artéria pulmonar, SvO2: saturação venosa mista, Na+: sódio, Cl-: cloro. Momento basal (BL), 30 minutos após o estabelecimento do choque (CH30), e 20 e 60 minutos após o início do tratamento (T20 e T60). Os dados são expressos como média \pm EP. ${ }^{*} p<0,05$ vs. BL.

No território esplâncnico, a hipovolemia esteve associada à redução de cerca de $75 \%$ do fluxo sangüíneo na veia porta, da oferta esplâncnica de oxigênio (107 \pm 15 para 22,3 $\pm 2,3 \mathrm{ml} / \mathrm{min}$ ), da saturação venosa de oxigênio do sangue porta (86,2 $\pm 2,2$ para $43,9 \pm 5 \%$ ) e do consumo esplâncnico de oxigênio (15,8 $\pm 1,3$ para $9,4 \pm 0,6 \mathrm{ml} / \mathrm{min})$; com aumento proporcional da taxa de extração esplâncnica de oxigênio de $16,4 \pm 2,1 \%$ para $57 \pm 5,2 \%(p<0,001)$ (Figura 2, Tabela 2). Foi observada, durante o estado de choque, uma redução significativa do $\mathrm{pH}$ intramucoso e concomitante aumento dos gradientes sistêmicos e esplâncnicos de $\mathrm{CO}_{2}$ analisados (Figura 3, Tabela 2).

A infusão da solução salina hipertônica promoveu restabelecimento parcial da pressão arterial média, do débito cardíaco e da saturação venosa mista de oxigênio. Observamos uma recuperação parcial, nos primeiros 20 minutos após o início do tratamento, do fluxo sangüíneo da veia porta; da oferta, consumo e taxa de extração esplâncnica de oxigênio. Os níveis de hemoglobina arterial sofreram uma redução de 14,9 $\pm 0,3$ para 9,9 $\pm 0,4 \mathrm{~g}$ /dl quando comparados ao momento basal $(p<0,05)$ (Tabela 1$)$. O pH intramucoso e os gradientes sistêmicos e esplâncnicos de $\mathrm{CO}_{2}$ apresentaram uma recuperação parcial de seus valores, entretanto não retornaram aos valores basais, mesmo após uma hora de observação (Figura 3). Não houve diferença da fração do débito cardíaco, direcionada para o território esplâncnico durante o choque ou após o início do tratamento (Tabela 2).

TABELA 2 - Variáveis hemodinâmicas e laboratoriais regionais durante o protocolo experimental

\begin{tabular}{lllll}
\hline & BL & CH30 & T20 & T60 \\
\hline FSVP (ml/min) & $504 \pm 73$ & $126 \pm 12^{*}$ & $318 \pm 32^{*}$ & $245 \pm 25^{*}$ \\
FSVP / DC (\%) & $26,8 \pm 2,8$ & $20,7 \pm 3,4$ & $28,7 \pm 2,3$ & $28,4 \pm 2,6$ \\
SpO2 (\%) & $86,2 \pm 2,3$ & $43,9 \pm 5^{*}$ & $72,4 \pm 3,2^{*}$ & $66,3 \pm 3,9^{*}$ \\
prCO2(mmHg) & $35,2 \pm 5$ & $74,7 \pm 6,7^{*}$ & $73 \pm 5,7^{*}$ & $64,6 \pm 4,9 *$ \\
pHi & $7,45 \pm 0,05$ & $6,98 \pm 0,05^{*}$ & $7,00 \pm 0,04^{*}$ & $7,08 \pm 0,02^{*}$ \\
Lactato portal $(\boldsymbol{\mu m o l} / \mathbf{L})$ & $0,9 \pm 0,2$ & $4,9 \pm 0,6^{*}$ & $4,7 \pm 0,6^{*}$ & $3,3 \pm 0,5^{*}$ \\
\hline
\end{tabular}

FSVP: Fluxo de sangue na veia porta, FSVP/DC: relação fluxo de sangue portal/débito cardiaco, SpO2: saturação venosa do sangue porta, prCO2: pressão parcial de dióxido de carbono na mucosa gástrica, pHi: pH intramucoso. Momento basal (BL), 30 minutos após oestabelecimento do choque (CH30), e 20 e 60 minutos após o início do tratamento (T20 e T60). Os dados são expressos como média \pm EP. ${ }^{*} p<0,05$ vs. BL.

FIGURA 2 - Variáveis derivadas do transporte de oxigênio sistêmico e esplâncnico durante o protocolo experimental. Momento basal (BL), 30 minutos após o estabelecimento do choque (CH30), e 20 e 60 minutos após o início do tratamento (T20 e T60). Os dados são expressos como média \pm EP. ${ }^{*} p<0,05$ vs. BL.
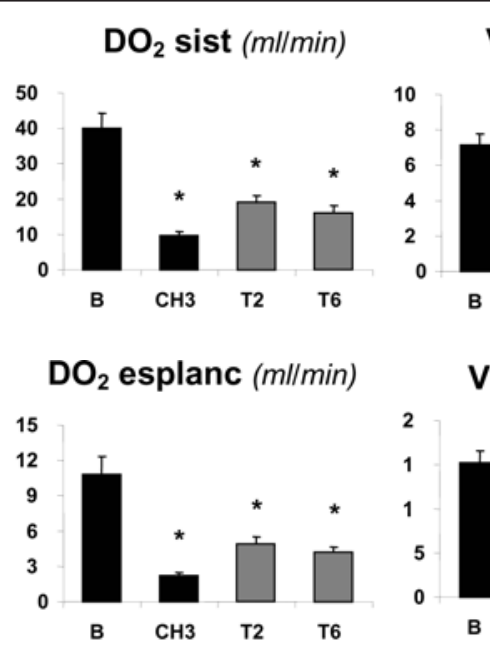

$$
\left.\mathrm{VO}_{2} \text { sist ( } \mathrm{m} / / \mathrm{min}\right)
$$

$\mathrm{TEO}_{2}$ sist (\%)
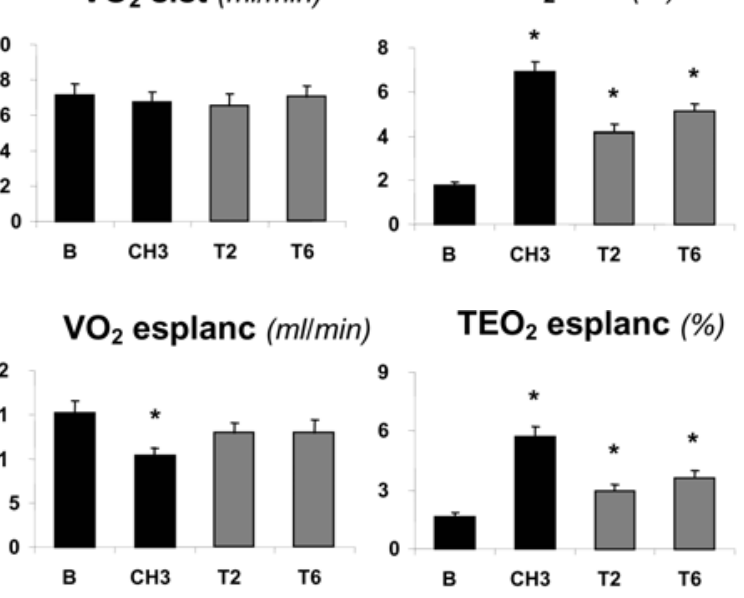

$\mathrm{TEO}_{2}$ esplanc (\%)

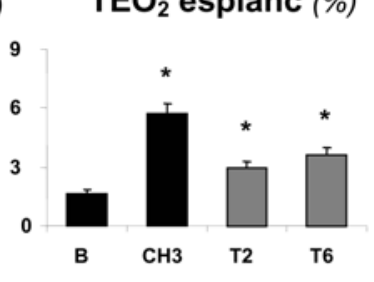


A concentração de sódio e cloro no sangue arterial apresentaram um aumento significativo, permanecendo inalterada mesmo após uma hora da infusão da SSH 7,5\% (Tabela 1). Uma correlação direta do $\mathrm{D}_{\mathrm{vp}-\mathrm{a}} \mathrm{pCO}_{2}$ e do $\mathrm{D}_{\text {ap-a }}$
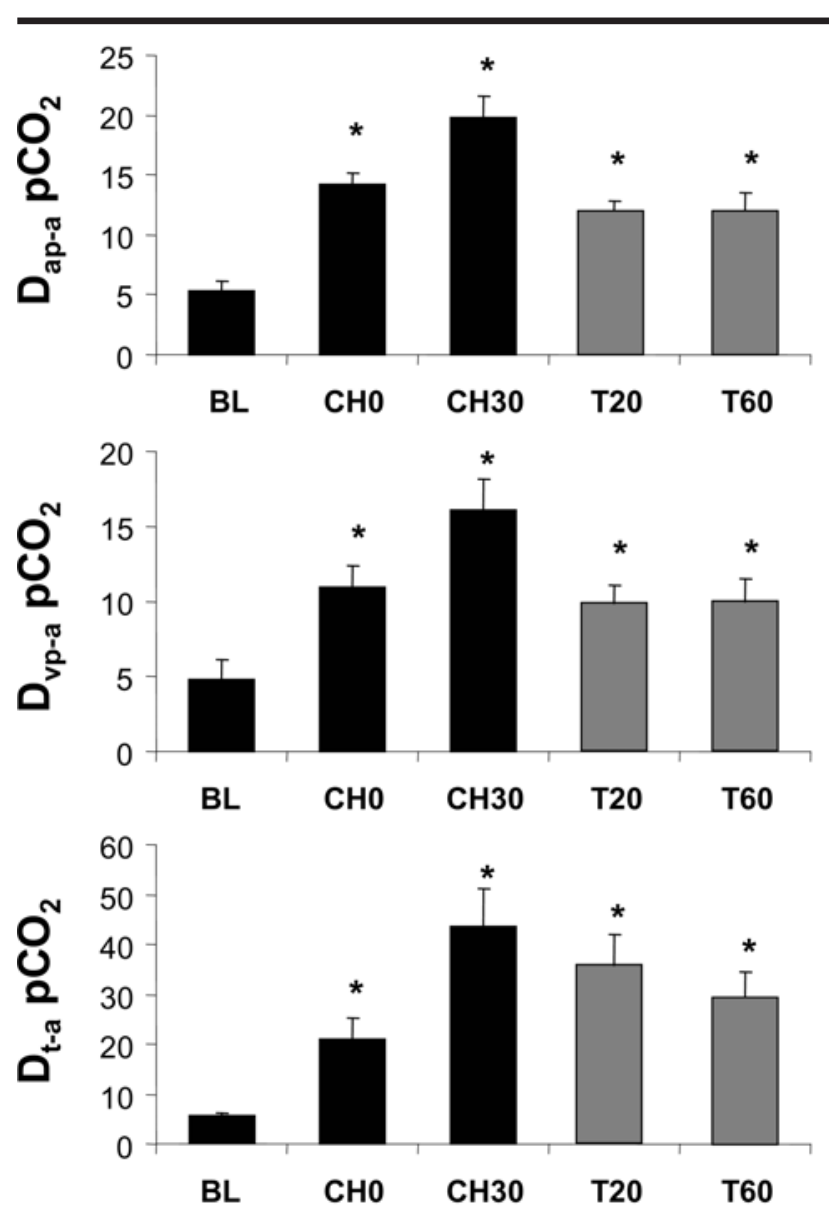

FIGURA 3 - Gradientes veno-arterial (Dap-apCO2), portaarterial (Dvp-apCO2) e mucosa-arterial (DtapCO2) de $\mathrm{CO} 2$ durante o protocolo experimental. Momento basal (BL), 30 minutos após o estabelecimento do choque (CH30), e 20 e 60 minutos após o início do tratamento (T20 e T60). Os dados são expressos como média \pm EP. $* p<0,05$ vs. BL.

\section{Discussão}

A hemorragia promoveu redução significativa do débito cardíaco, da pressão arterial média e do fluxo sangüíneo esplâncnico. Estas alterações foram acompanhadas por diminuição da saturação venosa mista, refletindo o aumento da taxa de extração sistêmica de oxigênio em resposta à redução da oferta de oxigênio, hiperlactatemia e consumo de base com conseqüente acidose metabólica. Fenômenos também evidenciados no território esplâncnico. Ainda foi observado aumento dos gradientes de $\mathrm{CO}_{2}$ veno-arterial, porta-arterial e gastroarterial, refletindo os graves distúrbios do fluxo sangüíneo induzidos pelo sangramento. A expansão volêmica com SSH, apesar de atenuar a hipotensão, a redução dos fluxos regional e sistêmico e a elevação dos gradientes de $\mathrm{CO}_{2}$, foi
$\mathrm{pCO}_{2}$ foi observada durante o período de choque e reposição volêmica $\left(R^{2}=0,6311\right)$. Porém, não houve correlação do $D_{t}$ ${ }_{\mathrm{a}} \mathrm{pCO}_{2}$ com os outros gradientes sistêmicos ou esplâncnicos de $\mathrm{CO}_{2}$ analisados ( $\mathrm{D}_{\mathrm{vp}-\mathrm{a}} \mathrm{pCO}_{2}$ e $\mathrm{D}_{\mathrm{ap}-\mathrm{a}} \mathrm{pCO}_{2}$; Figura 4).
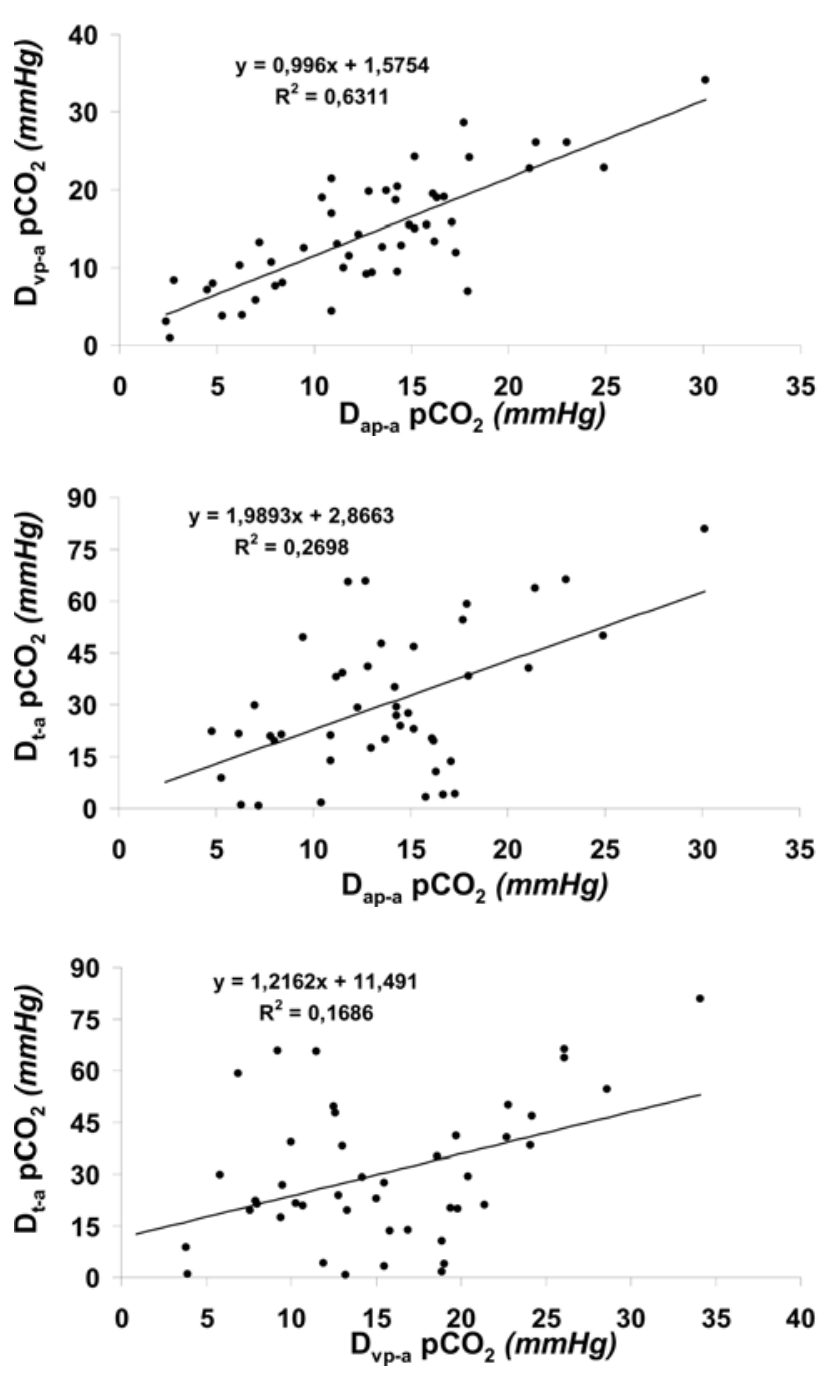

FIGURA 4 - Correlação entre os gradientes veno-arterial (Dap-apCO2), porta-arterial (Dvp-apCO2) e mucosa-arterial (Dt-apCO2) de CO2.

incapaz de reverter tanto os déficits perfusionais sistêmicos e/ou os regionais. A redução do fluxo sistêmico ocasionou uma queda proporcional do fluxo esplâncnico global enquanto o aumento da extração de oxigênio sistêmica e esplâncnica foram igualmente proporcionais. Este fenômeno também é descrito no choque por tamponamento pericárdico $^{13}$, mas não na $\operatorname{sepse}^{5}$, na qual o comprometimento regional é mais intenso sugerindo que no primeiro a reatividade vascular ainda está preservada, enquanto na sepse ocorre precocemente disfunção endotelial. $\mathrm{O}$ gradiente veno-arterial de $\mathrm{CO}_{2}$ acompanhou inversamente as alterações do débito cardíaco, enquanto o gradiente porta-arterial de $\mathrm{CO}_{2}$, acompanhou as alterações do fluxo porta, ambos apresentando um aumento significativo durante a fase de choque e redução durante a reanimação com SSH. A elevação destes gradientes tem sido 
demonstrado em estados de choque de diversas etiologias, sendo considerado importante marcador de hipoperfusão sistêmica e regional ${ }^{2,5,7,14,15}$.

Também foi observado aumento do $\mathrm{D}_{\mathrm{t}-\mathrm{a}} \mathrm{pCO}_{2}(6,1 \pm 1,1$ para $43,8 \pm 7,5 \mathrm{mmHg}$ ) o qual foi maior que o aumento do $\mathrm{D}_{\text {ap-a }} \mathrm{pCO}_{2}$ $(5,3 \pm 0,8$ para $19,9 \pm 1,6 \mathrm{mmHg})$ e do $\mathrm{D}_{\mathrm{vp-a}} \mathrm{pCO}_{2}(5,4 \pm 1,4$ para 22,6 $\pm 2,1 \mathrm{mmHg})$. Adicionalmente, foi detectado uma correlação direta entre o $\mathrm{D}_{\mathrm{ap}-\mathrm{a}} \mathrm{pCO}_{2}$ e o $\mathrm{D}_{\mathrm{vp}-\mathrm{a}} \mathrm{pCO}_{2}\left(\mathrm{r}^{2}=0,6311\right)$, mas não observado correlação entre $\mathrm{D}_{\mathrm{t}-\mathrm{a}} \mathrm{pCO}_{2}$ e tais gradientes. Diversos estudos clínicos e experimentais têm falhado em demonstrar uma boa correlação entre gradiente mucosa-arterial de $\mathrm{CO}_{2}$ e o fluxo sangüíneo hepatoesplâncnico ${ }^{2,4,15}$. A dissociação entre o comportamento do gradiente porta-arterial de $\mathrm{CO}_{2}$ com o $\mathrm{D}_{\mathrm{t}-\mathrm{p}} \mathrm{pCO}_{2}$ indica que, enquanto o primeiro acompanha as alterações circulatórias macro-regionais, o outro reflete apenas as alterações circulatórias e/ou metabólicas somente da mucosa gástrica, portanto, microcirculatórias. Estes dados sugerem que os distúrbios perfusionais macrocirculatórios regionais no choque hemorrágico podem ser estimados através da avaliação do gradiente sistêmico da $\mathrm{pCO}_{2}$, mas afirma a discrepância entre as alterações microcirculatórias e as macrocirculatórias.

A acidose da mucosa intestinal e conseqüente aumento do gradiente mucosa-arterial de $\mathrm{CO}_{2}$ pode estar associada a diversos mecanismos. Inicialmente estas alterações podem ser relacionadas a hipoxia tecidual e a ativação do metabolismo anaeróbico. Nestas situações de anerobiose, o aumento dos valores do dióxido de carbono ocorre devido ao tamponamento do excesso dos íons hidrogênio pelo bicarbonato. Além disso, em estados de hipoperfusão pode ocorrer acúmulo do $\mathrm{CO}_{2}$ gerado aerobicamente, devido à remoção inadequada deste metabólito ${ }^{7,16}$. Vandermeer et al., demonstraram que pode ocorrer acidose da camada mucosa na ausência de hipoxia e/ou hipoperfusão, sugerindo desordem do metabolismo de oxigênio em nível celular ${ }^{17}$.

Ao comparar a redução da oferta de oxigênio por redução do fluxo sangüíneo (hipóxia isquêmica) e por diminuição da $\mathrm{PaO}_{2}$ com fluxo sangüíneo constante (hipóxia hipóxica), Vallet et al. demonstraram que a redução da oferta de oxigênio a níveis críticos em ambos os modelos proporcionaram a elevação do gradiente veno-arterial de $\mathrm{CO}_{2}$ somente no grupo da hipóxia isquêmica ${ }^{16}$. Os autores concluíram que a ausência de alteração deste gradiente não exclui a presença de disóxia tecidual.

Em nosso estudo, o consumo regional de oxigênio foi comprometido sugerindo que apenas no território esplâncnico tenha sido atingida a oferta crítica de oxigênio. Desta forma podemos inferir que o aumento nos valores do $\mathrm{D}_{\mathrm{t}-\mathrm{a}} \mathrm{pCO}_{2}$ foi decorrente, não apenas da estagnação de $\mathrm{CO}_{2}$ na camada mucosa, mais também da produção anaeróbica de $\mathrm{CO}_{2}$, visto que a redução do fluxo regional foi capaz de atingir a $\mathrm{DO}_{2}$ crítica. Alem disso ouma elevação significativa dos niveis de lactato esplâncnico foram observados.

A mucosa intestinal é a camada metabolicamente mais ativa e especialmente suscetível à isquemia decorrente das alterações do fluxo sangüíneo sistêmico. A vasta inervação simpática, ativada com a redução sistêmica da $\mathrm{DO}_{2}$, associada à ativação do sistema renina-angiotensina, promovem vasoconstricção esplâncnica e conseqüente redistribuição do fluxo sangüíneo para a circulação central. As características da arquitetura microvascular intestinal também contribuem para esta suscetibilidade. A arteríola do vilo forma um ângulo reto com a artéria mesentérica, que resulta em redução do hematócrito no vilo ("plasma skimming”). Além disso, a proximidade entre a arteríola e a vênula permite a formação de mecanismo de contra-corrente, promovendo redução progressiva da pressão parcial de oxigênio da base para o ápice do vilo. Portanto, em condições fisiológicas, o vilo intestinal tende a ser relativamente hipóxico mesmo em repouso 2,3,5,18.

Estudos experimentais demonstram que o fluxo sangüíneo no território esplâncnico, particularmente na mucosa gastrointestinal, é precoce e intensamente comprometido nos quadros de hipoperfusão sistêmica ${ }^{2-5}$. Em voluntários saudáveis submetidos a retirada gradual de $25 \%$ da volemia, a tonometria gástrica detectou redução do fluxo sangüíneo regional antes que as variáveis sistêmicas clínicas e laboratoriais habitualmente utilizadas, como freqüência cardíaca, pressão arterial, lactato, excesso de base, apresentassem alterações ${ }^{19}$.

Em adição à suscetibilidade da camada mucosa à hipóxia, durante a fase de ressuscitação em diversos estados de choque, a recuperação do fluxo no território esplâncnico é ulterior e a hipoperfusão regional pode persistir a despeito da normalização ou mesmo supranormalização das variáveis sistêmicas ${ }^{2-4,15,19}$. Esta hipoperfusão persistente tem sido associada à disfunção da barreira mucosa, sepse e disfunção de múltiplos órgãos ${ }^{1,3,6}$. Embora a expansão com SSH não tenha sido capaz de restaurar os déficits perfusionais, esta atenuou a queda dos fluxos sistêmico e regional assim como a hipotensão, ambos eventos associados à disfunção orgânica e mortalidade. Desta forma, a expansão volêmica proporcionada pela SSH foi benéfica como intervenção inicial. Deve ser considerado que foi administrado apenas $16 \%$ do volume sangrado em 5 minutos sendo que seus benefícios foram observados até 1 hora após início do fluído. Período em que outras medidas adicionais podem ser implementadas para restabelecer o transporte de oxigênio e normalizar o metabolismo celular. A restauração do fluxo sangüíneo em vários territórios, inclusive o esplâncnico, está associada à lesão de isquemia e reperfusão. Durante a fase de reperfusão, além da formação de radicais livres, ocorre a liberação de mediadores inflamatórios que promovem ativação e aderência de leucócitos no endotélio, com conseqüente comprometimento do fluxo na microcirculação, apesar do restabelecimento do fluxo nos grande vasos, fenômeno denominado no-reflow ${ }^{11}$. A favor desta hipótese podemos observar que o pré-tratamento com anti-oxidantes, como a glutadiona ou mesmo o bloqueio da enzima xantina oxidase, promove uma proteção significativa contra a lesão de reperfusão gástrica após episódio de isquemia esplâncnica induzida pelo choque hemorrágico ou oclusão da aorta abdominal ${ }^{20}$.

O efeito inicial da injeção de $4 \mathrm{ml} / \mathrm{kg}$ de $\mathrm{NaCl}$ a $7.5 \%$ (2400 mOsm/l) é físico, ou seja, ocorre uma expansão do volume circulatório devido ao gradiente osmótico criado desviando a água para o compartimento intravascular. As principais fontes para a expansão plasmática observada são as hemácias e o endotélio, que estão em íntimo contato com a hipertonicidade plasmática, perdendo cerca de $8 \%$ de seus volumes para o compartimento intravascular. Além da expansão volêmica, este fato produz importantes conseqüências hemodinâmicas, pois o edema de hemácias e das células endoteliais, observados no choque e na isquemia-reperfusão, tem importância crítica em 
termos de viscosidade e de resistência hidráulica da microcirculação, local onde a razão entre a luz do vaso e o tamanho da hemácia aproximase da unidade. Nestas circunstâncias, o edema celular compromete o fluxo sangüíneo microcirculatório em diversos territórios, e a solução hipertônica, mas não a isotônica, contribui para a recuperação da hemodinâmica na microcirculação ${ }^{11}$.

Desta forma o objetivo deste estudo foi testar a hipótese que a reposição volêmica inicial com SSH poderia trazer benefícios adicionais à perfusão da mucosa do trato digestivo. Entretanto, ao contrario da nossa hipótese não pudemos observar a restauração completa do fluxo esplâncnico global após a infusão da SSH.Alguns fatores podem explicar nossos achados e constituem as principais limitações do estudo. Inicialmente, no modelo de choque utilizado houve retirada de aproximadamente $43 \%$ da volemia total, correspondendo a um estado de choque classe IV, onde é imperativa a utilização de sangue e hemoderivados entretanto, não foi administrado volumes adicionais de cristalóides, colóides ou mesmo hemoderivados. Além disso, o seguimento dos animais após a reposição volêmica foi extremamente curto impedindo avaliar o impacto das alterações perfusionais na disfunção de múltiplos órgãos e na sobrevida dos animais.

\section{Conclusão}

A administração de SSH promoveu benefícios parciais na perfusão sistêmica e esplâncnica, os quais foram especialmente limitados na microcirculação regional, evidenciando a elevada susceptibilidade da mucosa gastrintestinal a fenômenos de isquemiareperfusão e sua ulterior recuperação durante a fase de ressuscitação. Ainda, observamos que as variáveis sistêmicas e regionais dependentes de oxigênio, não refletem a adequação da perfusão da mucosa gástrica nos estado de choque, enfatizando a importância da monitorização deste território com a finalidade de otimizar as manobras de reanimação, podendo desta forma minimizar a morbidade e mortalidade do paciente politraumatizado.

\section{Referências}

1. Kreimeier U. Pathophysiology of fluid imbalance. Crit Care. 2000;4(Suppl 2):S3-7.

2. Cruz RJ, Yada-Langui MM, Poli de Figueiredo LF, Rocha e Silva $M$. Effects of hemorrage and rapid fluid resuscitation on splanchnic blood flow and gastrointestinal mucosal perfusion evaluated by gas tonometry. ABCD Arq Bras Cir Dig. 2002;15:74-8.

3. Poli De Figueiredo LS, E; Cruz Junior, RJ; Rocha E Silva, M. Gas tonometry for evaluation of gastrointestinal perfusion: experimental models of trauma, shock and complex surgical maneuvers. Part 1. Acta Cir Bras. 2002;17:211-9.

4. Lagoa CE, De Figueiredo LF, Cruz RJ, Jr., Silva E, Rocha ESM. Effects of volume resuscitation on splanchnic perfusion in canine model of severe sepsis induced by live Escherichia coli infusion. Crit Care. 2004;8:R221-8.

5. Garrido AG, Poli de Figueiredo LF, Cruz Jr RJ, Silva E, Rocha ESM. Short-lasting systemic and regional benefits of early crystalloid infusion after intravenous inoculation of dogs with live Escherichia coli. Braz J Med Biol Res. 2005;38:873-84.

6. Marik PE. Gastric intramucosal pH. A better predictor of multiorgan dysfunction syndrome and death than oxygen-derived variables in patients with sepsis. Chest. 1993;104:225-9.

7. Weil MH, Sun S. Tissue capnometry. Crit Care Med. 2001;29:460.

8. Ronholm E, Runeborg J, Karlsen KL, Tomasdottir H, Aneman A, Bengtsson A. Perioperative gastric tonometric PCO2 and intramucosal $\mathrm{pH}$ in patients undergoing liver transplantation. Acta Anaesthesiol Scand. 1999;43:695-701.

9. Ivatury RR, Simon RJ, Islam S, Fueg A, Rohman M, Stahl WM. A prospective randomized study of end points of resuscitation after major trauma: global oxygen transport indices versus organspecific gastric mucosal pH. J Am Coll Surg. 1996;183:145-54.

10.Alam HB, Stanton K, Koustova E, Burris D, Rich N, Rhee P. Effect of different resuscitation strategies on neutrophil activation in a swine model of hemorrhagic shock. Resuscitation. 2004;60:919.

11.Rocha e Silva M, Poli de Figueiredo LF. Small volume hypertonic resuscitation of circulatory shock. Clinics. 2005;60:159-72.

12.Fiddian-Green RG, Pittenger G, Whitehouse WM, Jr. Backdiffusion of $\mathrm{CO} 2$ and its influence on the intramural $\mathrm{pH}$ in gastric mucosa. J Surg Res. 1982;33:39-48.

13.Zhang H, Smail N, Cabral A, Cherkaoui S, Peny MO, Vincent JL. Hepato-splanchnic blood flow and oxygen extraction capabilities during experimental tamponade: effects of endotoxin. J Surg Res. 1999;81:129-38.

14.Maynard N, Bihari D, Beale R, Smithies M, Baldock G, Mason $\mathrm{R}$, McColl I. Assessment of splanchnic oxygenation by gastric tonometry in patients with acute circulatory failure. J Am Med Assoc. 1993;270:1203-10.

15. Oud L, Kruse JA. Progressive gastric intramucosal acidosis follows resuscitation from hemorrhagic shock. Shock. 1996;6:615.

16. Vallet B, Teboul JL, Cain S, Curtis S. Venoarterial CO(2) difference during regional ischemic or hypoxic hypoxia. J Appl Physiol. 2000;89:1317-21.

17. VanderMeer TJ, Wang H, Fink MP. Endotoxemia causes ileal mucosal acidosis in the absence of mucosal hypoxia in a normodynamic porcine model of septic shock. Crit Care Med. 1995;23:1217-26.

18.Cruz RJ, Jr., de Figueiredo LF, Braz JL, Diniz EA, Rocha e Silva M. Systemic and regional effects of supraceliac aortic occlusion during experimental hepatic vascular exclusion. Am J Surg. 2003;185:388-93.

19.Hamilton-Davies C, Mythen MG Salmon JB, Jacobson D, Shukla A, Webb AR. Comparison of commonly used clinical indicators of hypovolaemia with gastrointestinal tonometry. Int Care Med. 1997;23:276-81.

20.Nielsen VG, Tan S, Baird MS, McCammon AT, Parks DA. Gastric intramucosal pH and multiple organ injury: impact of ischemia-reperfusion and xanthine oxidase. Crit Care Med. 1996;24:1339-44.
Correspondência:

Ruy J. Cruz Junior

Rua Marivaldo Fernandes, 140

04792-060 São Paulo - SP Brasil

expcruzjr@incor.usp.br
Conflito de interesse: nenhum

Fonte de financiamento: FAPESP (Proc 98/15658-0)

Recebimento: 19/10/2005

Revisão: 21/11/2005

Aprovação: 19/12/2005

\section{Como citar este artigo:}

Cruz Jr RJ, Yada-Langui MM, Garrido AG, Figueiredo LFP, Silva MR. Efeitos iniciais da reposição volêmica com solução salina hipertônica a 7,5\% na perfusão e oxigenação esplâncnica após choque hemorrágico. Acta Cir Bras. [periódico na Internet] 2006 MarAbr;21(2). Disponível em URL: http://www.scielo.br/acb 